\title{
ADDICIONES Y CORRECCIONES A LA MONOGRAFÍA RUBIÁCEAS DE CUBA
}

\author{
A. L. Borhidi ${ }^{1}$, M. Fernández Zequeira ${ }^{2}$ y R. Oviedo-Prieto ${ }^{2}$ \\ ${ }^{1}$ Institute of Biology, University of Pécs, H-7624 Pécs, Ifjúság útja 6, Hungary \\ E-mail: borhidi@gamma.ttk.pte.hu \\ ${ }^{2}$ Instituto de Ecología y Sistemática, Ministerio de Ciencia, Tecnología y Medio Ambiente \\ La Habana, Cuba
}

(Received 26 June, 2018; Accepted 31 July, 2018)

\begin{abstract}
An important molecular-phylogenetic monography appeared about the Chiococceae tribe of the Caribbean region by Paudyal et al. (2018) proposing several new taxonomic modifying decisions concerning also to the monographic treatment of the recently (2017) published Rubiáceas de Cuba, as the confirmation and extension of the genus Solenandra revalidated by Borhidi (2002) and the separation of the new endemic Cuban genus Ramonadoxa Paudyal and Delprete from Chiococca. The molecular-phylogenetic studies did not verify the separation of Ceuthocarpus Aiello from Schmidtottia Urb. neither Phyllacanthus Hook. f. as independent genus from Catesbaea $\mathrm{L}$.
\end{abstract}

Key words: Catesbaea, Chiococca, Exostema, Ramonadoxa, Rubiáceas de Cuba, Schmidtottia, Solenandra

\section{INTRODUCCIÓN}

En noviembre del año 2017 se publicó el tratamiento monográfico de la familia Rubiaceae, teniendo en cuenta la contribución al proyecto en desarrollo Flora de la República de Cuba, basado en investigaciones morfológicas y taxonómicas realizadas durante los últimos 45 años por los autores de este artículo, utilizando también los numerosos aportes importantes de difunto Lic. Onaney Muñiz Gutierrez, del Dr. Miguel Angel Vales García, la Lic. Milagros Moncada, el Dr. René Pablo Capote López, el Dr. Pedro Herrera Oliver y de muchos otros colegas (véase la página 460 del libro Rubiáceas de Cuba). En el caso de algunos aspectos taxonómicos discutidos basamos nuestras decisiones en los conocimientos morfológicos y al no haber podido tomar en consideracion los argumentos filogenéticos-molecular todavía desconocidos para muchos taxones. Este vacío de conocimientos ha sido ocupado en parte por el reciente y valioso estudio molecular realizado en 27 géneros y 125 especies de la tribu Chiococceae del Caribe por Paudyal et al. (2018) que tuvo como resultado la descripción de 4 géneros nuevos y muchas combinaciones nuevas para la ciencia. Las propuestas taxonómicas nuevas para la flora de Cuba son las siguientes. 


\section{DISCUSIÓN}

\section{Catesbaea y Phyllacanthus}

Según los análisis moleculares realizados por Paudyal et al. para estos dos géneros tratados como separados por varios autores (Alain 1962, Borhidi et al. 2017) no resultaron ser dos géneros distintos, aunque tienen caracteres morfológicos muy vistosos (véase Borhidi et al. 2017 fig. 11. y fig. 84). Aceptando las sugerencias de Paudyal et al. la clave y la relación de especies de Catesbaea L. para Cuba se modifican como sigue.

\section{CATESBAEA L., 1753}

Catesbaea L., Sp. Pl. 109 (1753). - Typus generis: Catesbaea spinosa L. - Syn.: Phyllacanthus Hook. f.

Arbustos o arbustillos, a veces arbolitos o trepadores, glabros o pubescentes, comúnmente armados por espinas largas. Hojas pequeñas opuestas o a menudo fasciculadas, a veces áfilos o casí con ramas rígidas, divaricadas y espinas muy grandes, semi-deltoideas, verticalmente comprimidas y las hojas escamiformes, diminutas, caducas; estípulas interpeciolares, pequeñas, caedizas. Flores axilares, solitarias, pediceladas; lóbulos del cáliz 4, rara vez 5 a 6 , subulados, persistentes. Corola embudada o acampanada, la garganta glabra, lóbulos 4, rara vez 5 a 6, cortos, valvares. Estambres 4, insertos en la base de la corola; filamentos filiformes, anteras dorsifijas cerca de la base, lineares, 2-lobuladas en la base. Ovario 2-locular, estilo filiforme, estigma alargado. Óvulos numerosos o pocos. Fruto comúnmente pequeño, ovoide o globoso, 2-locular. Semillas numerosas y comprimidas o pocas angulosas, endospermo carnoso.

Género endémico de las Antillas y Bahamas con unas 30 espécies; en Cuba 10 especies de ellas 8 endémicas.

1a Tallo comprimido, alado, espinoso-lobulado, áfilo o las hojas escamiformes, decíduas

9. C. phyllacantha

1b Tallo cilíndrico, no alado, espinoso en los nudos, hojas existentes 2

2a Corola de 2 a $20 \mathrm{~cm}$; fruto a menudo de $2 \mathrm{~cm}$ de diámetro o más 3

2b Corola y fruto de hasta 1,5 cm de largo

3a Corola de $2 \mathrm{~cm}$; hojas aovado-triangulares

1. C. flaviflora

3b Corola de 5 a $20 \mathrm{~cm}$ de largo 
4a Corola de 5 a $7 \mathrm{~cm}$ de largo

5. C. longispina

$4 \mathrm{~b}$ Corola de $10 \mathrm{a} 20 \mathrm{~cm}$ de largo

10. C. spinosa

5a Fruto de $1,5 \mathrm{~cm}$ de diámetro

6. C. macracantha

5b Fruto de 2 a $13 \mathrm{~mm}$ de diámetro

6a Óvulos 14 a 17 en cada celda del ovario

3. C. grayi

6b Óvulos 1 a 6 en cada celda del ovario 7

7a Óvulo solitario en cada celda del ovario

2. C. gamboana

$7 \mathrm{~b}$ Óvulos 2 a 6 en cada celda del ovario

8a Fruto de 4 a $7 \mathrm{~mm}$ de diámetro, rojo; espinas de hasta $12 \mathrm{~mm} \quad$ 7. C. nana

$8 \mathrm{~b}$ Fruto de 2 a $4,5 \mathrm{~mm}$ de diámetro; espinas de hasta $25 \mathrm{~mm}$ 9

9a Fruto blanco, 3 a 4,5 $\mathrm{mm}$ de diámetro; hojas de 3 a $15 \mathrm{~mm}$ de largo

8. C. parviflora

9b Fruto rojo, 2 a $3 \mathrm{~mm}$ de diámetro; hojas de 1 a $5 \mathrm{~mm}$ de largo

4. C. holoacantha

1. Catesbaea flaviflora Urb., Symb. Ant. 9: 157 (1923). - Tipo: Cuba. Oriente, prope Santiago de Cuba, in declivibus calcareis, siccis. Leg.: Ekman (7731). Holotipo: Bt; lectotipo: S!.

2. Catesbaea gamboana Urb., Symb. Ant. 9: 524 (1928). - Tipo: Cuba, Oriente, Gamboa. Leg.: Ekman (14950). Holotipo: B十; lectotipo: S!.

3. Catesbaea grayi Griseb., Pl. Wr. Mem. Acad. Amer. 8: 503 (1863). - Tipo: Cuba, Or.: Monte Verde. Leg.: Wright (371). Holotipo: GOET; isotipo: GH!.

4. Catesbaea holoacantha Wr. ex Griseb., Cat. Pl. Cub. 122 (1866). - Tipo: Cuba, Or.: Mayari abajo. Leg.: Wright (2657). Holotipo: GOET; isotipo: GH n.v.

5. Catesbaea longispina A. Rich. in Sagra, Hist. Cuba 11: 12 (1850). Tipo: Cuba; Cienfuegos, La Jagua, Ossa s.n. Holotipo: P!.

6. Catesbaea macracantha Wr. in Sauv., Anal. Acad. Ci. Habana 6: 99 (1869). - Tipo: Cuba. Oriente, Potrero Manatí. Leg.: Wright (3575). Holotipo: $\mathrm{GH}$; isotipo: HAC!.

7. Catesbaea nana Greenm. ex Combs, Trans. Acad. St. Louis 7: 428 (1897). - Tipo: Cuba, Prov. Cienfuegos, Cienaguita. Leg.: Combs 271 Bt; F.

8. Catesbaea parviflora Sw., Prodr., p. 30 (1788). = Scolosanthus parviflorus (Sw.) C. Wright in Sauv., Anal. Acad. Ci. Habana 6: 99 (1869). - Tipo: Jamaica. Leg.: Swartz s.n. Holotipo: S!. - Syn.: Catesbaea campanulata Sagra ex DC., Prodr. 4: 401 (1830). E Echinodendron campanulatum A Rich. in Sagra, Hist. Cuba 11: 18 (1850). 
9. Catesbaea phyllacantha Griseb., Cat. Pl. Cub. 122 (1866). - Basionym: Phyllacanthus grisebachianus Hook. f., Hooker's Icon. Pl. 11: 77, tab. 1095 (1871). - Tipo: Cuba. 'Cultivated but indigenous on the north coast'. Leg.: C. Wright (2655), 1863. Holotipo: GOET; isotipos: K, MO, NY, S.

10. Catesbaea spinosa L., Sp. Pl., p. 109 (1753). - Tipo: basado en Catesby: Nat. Hist. Carolina, p. 100., tab. 100. (1730-1748). - Syn.: Catesbaea macrantha A Rich. in Sagra, Hist. Fis. Pol. Nat. Cub. 11: 12 (1850). = Catesbaea longiflora Sw. Prodr. 1788. $=$ Catesbaea latifolia Lindl., Bot. Reg. t. 858.

Destacamos: que una de las especies incluídas en el análisis molecular, la Catesbaea phyllacantha Griseb. es una especie extinguida por la destrucción del área de Toscano, Provincia Pinar del Rio, su localidad clásica y por lo que no ha sido recolectada desde hace casi 100 años, mientras que las demás especies incluídas en el análisis molecular fueron analizadas a partir de material fresco. Por eso, es posible, que en la comparación las secuencias de las bases no fueron iguales para el caso de las especies de Catesbaea y de Phyllacanthus.

\section{Ceuthocarpus y Schmidtottia}

El género endémico serpentinícola Ceuthocarpus fue descrito por Aiello en 1979, basado en Portlandia involucrata Wernh. (1913) transferida posteriormente al género Schmidtottia Urb. por Alain (1959). Los caracteres distintivos de Ceutocarpus determinados por Aiello fueron la flor terminal rodeada por un involucro de 3-4 hojas y los estambres exertos de la garganta de la flor, mientras en las especies del género Schmidtottia las flores no están involucradas y los estambres están incluídos en el tubo de la corola. Borhidi (2002) encontró, que las poblaciones de Ceuthocarpus involucratus tienen una variación notable determinada por las condiciones ecológicas diversas de las distintas areas de serpentina en las montañas del Norte de Oriente, y distinguió una raza del Río Toa como la variedad típica (var. involucrata), la raza de Moa (var. moaensis) y la raza de Maraví-Baracoa (var. elatior). Los estudios moleculares de Paudyal et al. (2018) no encontraron diferencias notables entre las secuencias básicas de Ceuthocarpus y de las especies del género Schmidtottia y no confirmaron su posición taxonómica como género separado. Los autores de la monografía de Rubiáceas de Cuba siguen la sugerencia de Paudyal et al. (2018) y tratan el género Ceuthocarpus incluido en Schmidtotta, modificando también la posición taxonómica de las unidades subespecificas descritas originalmente bajo Ceuthocarpus. En consecuencia la clave y la relación de las especies cambian de la siguiente forma: 


\section{SCHMIDTOTTIA Urb., 1923}

Schmidtottia Urb., Symbolae Antillanae 9: 143 (1923). - Tipo: S. monantha Urb., Symb. Antill. 9: 138 (1923). Syn.: Ceuthocarpus Aiello, J. Arnold Arbor. 60: 109 (1979).

Arbustos o arbolitos, las ramas mayormente resinosas. Estípulas connadas en una vaina truncada. Hojas opuestas o en verticilos de a 3. Inflorescencias terminales, 1-multifloras, acabezueladas, cimoso-corimbosas o apanojadas. Flores vistosas, amarillas, rosadas a rojas o purpureas, 5-meras. Cáliz con tubo turbinado, lóbulos lineares, lanceolados o triangulares, persistentes. Corola 5-angulosa, lóbulos induplicado-imbricados, relativamente cortos, agudos y erguidos. Estambres sublibres de la corola, filamentos connados en la base, muy brevemente pelosos abajo, glabros arriba; anteras dorsifijas arriba de la base, lineares, obtusas en el ápice. Ovario infero, 2-locular placenta oval $\mathrm{u}$ obovada, al centro del septo peltadamente fija, óvulos numerosos. Estilo entero o 2-!obulado, glabro. Cápsula obovada a obovado-turbinada, angulosa, de dehiscencia primeramente septicida; placenta central con 10-16 semillas sentadas, algunas ascendentes otras péndulas, sin apéndice, embrión pequeño.

Género endémico Cubano con 17 especies.

\section{Clave para las especies}

1a Flores rodeadas por un involucro de brácteas foliáceas; estambres exertos

5. S. involucrata

1b Flores no rodeadas por un involucro; estambres insertos 2

2a Hojas sésiles 3

2b Hojas pecioladas $\quad 8$

3a Flores en capítulos, estos sésiles o subsésiles 4

$\begin{array}{lll}3 \mathrm{~b} & \text { Flores pediceladas en umbelas paucifloras } & 7\end{array}$

4a Ramitas e hipantio pubérulos a hirsutos, hojas de 1,5-4 cm, corola tubular, de 3-4 mm de ancho, pubérula

4b Ramitas e hipantio glabros por fuera, corola estrechamente acampanulada, de 10-14 mm de ancho, glabra, hojas de 3-7 cm

5a Hojas escabrosas en el haz; lóbulos del cáliz 5, hirsutos, corola de 1,5 cm de largo

14. S. sessiliflora

5b Hojas glabras y lisas en el haz, lóbulos del cáliz 4, glabros; corola 22,5 cm de largo

8. S. monticola 
6a Lóbulos del cáliz lineales, de 4-5 mm de largo, reflejos, cápsula 4-angular, de 7-10 mm de largo

10. S. neglecta

6b Lóbulos del cáliz triangulares de 1-2 mm de largo, rectos, cápsula de 10-12 mm de largo, 10-acostillado

15. S. shaferi

7a Hojas de 2-5 cm de largo, escabrosas y mates en ambas caras; corola amarilla, de 1,5-2 cm de largo

13. S. scabra

7b Hojas de 4-10 cm de largo, brillantes y lisas en ambas caras; corola roja, de $2,5 \mathrm{~cm}$ de largo

11. S. nitens

8a Flores en corimbos o panojas 3-multifloras 9

8b Flores mayormente solitarias, terminales, grandes 14

9a Lóbulos del cáliz planos, ensanchados o cuculados, no subulados 10

9b Lóbulos del caliz involutos, subulados

10a Lóbulos del cáliz ensanchados y cuculados en el ápice $\quad 3$. S. cucullata

10b Lóbulos del cáliz no cuculados en el ápice

11a Lóbulos del cáliz ensanchados en el ápice

6. S. marmorata

11b Lóbulos del cáliz lineari-oblongos, estrechados en el ápice obtuso 12

12a Inflorescencia corimbiforme, pedicelos muy cortos de 0-2 $\mathrm{mm}$ de largo

9. S. multiflora

12b Inflorescencia apanojada, laxa, pedicelos de 0,5-2 cm de largo

4. S. elliptica

13a Inflorescencia 2-4-flora, cáliz peloso, corola de 3-4 cm; cápsula de 7-10 mm de largo

1. S. corymbosa

13b Inflorescencia apanojada, multiflora, cáliz glabro, corola de $2-2,5 \mathrm{~cm}$, cápsula de $12-18 \mathrm{~mm}$ de largo

16. S. stricta

14a Hojas elíptico-oblongas a oblongo-lanceoladas, atenuadas en el ápice, mayormente en verticilos de 3-4

2. S. cubensis

14aa Hojas escabrosas en el haz, flores de 2,5-3,5 cm de largo

2b. ssp.cubensis

14bbHojas lisas, flores de 3,5-4,5 cm de largo

2a. ssp. cristalensis

14b Hojas opuestas, obovadas u ovales a suborbiculares, redondeadas en el ápice 
15a Hojas elípticas a suborbiculares de 6-10 mm de largo, redondeadas a obtusas en la base

12. S. parvifolia

15b Hojas obovadas de 1-3 cm de largo, estrechadas a agudas en la base 16

16a Hojas escabrosas a glabrescentes en el haz, flor de 4-4,5 cm de largo, cápsula de 8-9 mm de largo y 3,5-4 $\mathrm{mm}$ de ancho en el ápice

7. S. monantha

16b Hojas glabras en el haz, flor de $2-3 \mathrm{~cm}$ de largo, cápsula de 1,5 cm de largo y $6 \mathrm{~mm}$ de ancho en el ápice

17. S. uliginosa

1. Schmidtottia corymbosa Borhidi in Borhidi et al., Abstracta Bot. 5: 37 (1977). - Tipo: Cuba, Prov. Oriente, camino de bajada a Coco, Mina Franklyn, Moa. Leg.: León, Clemente y Néstor, León (23224), 20.07.1947. Holotipo: HAC!; isotipos: HAC!, NY! (Clemente 5543).

2. Schmidtottia cubensis (Standl.) Urb., Symb. Antill. 9: 139 (1923). - Basionym: Isidorea? cubensis Standl., N. Am. Fl. 32: 15 (1918). - Tipo: Cuba, Sierra de Nipe; Arroyo del Medio above the falls, 450-500 m alt. Leg.: A. Shafer (3230), 20.01.1910. Holotipo: NY!; isotipo: HAC!.

\section{2a. Schmidtottia cubensis subsp. cubensis}

2b. Schmidtottia cubensis subsp. cristalensis (Borhidi et Muñiz) Borhidi, Acta Bot, Hung. 44: 56 (2002). - Basionym: Schmidtottia cubensis (Standl.) Urb. var. cristalensis Borhidi et Muñiz, Acta Bot. Acad. Sci. Hung. 21: 230 (1975). - Tipo: Cuba, Prov. Oriente, Sierra del Cristal: charrascos de La Loma El Serrucho. Leg.: Alain, Acuña y M. López Figueiras, Alain (5402), 2-7.04.1956. Holotipo: HAC; isotipos: HAC, NY.

3. Schmidtottia cucullata Borhidi et Bisse in Borhidi, Acta Bot. Acad. Sci. Hung. 26: 272 (1980). - Tipo: Cuba, Prov. Oriente, Moa, Sierra de Moa, Cayo Coco, charrascales en 200-300 m. alt. Leg.: Bisse y Lippold (17618), 13.08.1970. Holotipo: HAJB; isotipo: JE.

4. Schmidtottia elliptica (Britt.) Urb., Symb. Antill. 9: 139 (1923).

4a. Schmidtottia elliptica subsp. elliptica - Basionym: Portlandia elliptica Britt., Bull. Torr. Bot. Club 41: 22 (1914). - Tipo: Cuba, Prov. Oriente, Baracoa to Florida, thickets. Leg.: A. Shafer (4332), 15.03.1910. Holotipo: NY!; isotipo: HAC!.

4b. Schmidtottia elliptica subsp. oblongata Borhidi, Acta Bot. Acad. Sci. Hung. 26: 273 (1980). - Tipo: Cuba, Prov. Oriente, Moa; Cayoguán, camino hacía Mina Delta. Holotipo: HAC (Clemente 6829); isotipo: HAC (Alain 929).

5. Schmidtottia involucrata (Wernham) Alain, Contr. Ocas. Mus. Hist. Nat. Col. de la Salle 17: 10 (1959). - Basionym: Portlandia involucrata Wernham, J. Bot. 51: 320 (1913). झ Ceuthocarpus involucratus (Wernham) Aiello, J. Arnold Arbor. 60: 109 (1979). - Tipo: Cuba, Oriente: Moa, across Sierra Moa to Moa 
Bay, Camp La Gloria. Leg.: J. A. Shafer (8282), 31 Dec 1910-1 Jan 1911 (fr). Lectotipo: NY, designado por Aiello (1979: 109); isotipo: A.

5a. Schmidtottia involucrata var. involucrata

5b. Schmidtottia involucrata var. moaensis (Borhidi) Borhidi, comb. nova, hoc loco - Basionym: Ceuthocarpus involucratus Aiello var. moaensis Borhidi, Acta Bot. Hung. 44: 51 (2002). - Tipo: Cuba Oriental, Moa, orillas del Rio Cayoguán, cerca de la Mina. Leg.: Clemente, Nestor y Crisógone (4485), 02.07.1945. Holotipo: HAC!; isotipo NY!.

5c. Schmidtottia involucrata var. elatior (Borhidi) Borhidi, comb. nova, hoc loco - Basionym: Ceuthocarpus involucratus Aiello var. elatior Borhidi, Acta Bot. Hung. 44: 51 (2002). - Tipo: Cuba Oriental, Baracoa, charrascos en el valle del Rio Maraví. Leg. J. Bisse (18240). Holotipo: HAJB!.

6. Schmidtottia marmorata Urb., Symb. Antill. 9: 140 (1923). - Tipo: Cuba, Prov. Oriente, arbusto en charrascales del Rio Toa, cerca de Baracoa. Leg.: E. L. Ekman (3675); 28.11.1914. Holotipo: Bt; lectotipo: S!; isolectotipo: HAC!.

7. Schmidtottia monantha Urb., Symb. Antill. 9: 138 (1923). - Tipo: Cuba, Prov. Oriente, pinares del Rio Maraví, m. Dec. flor. et fruct. Leg. E. L. Ekman (4028), 25.12.1914. Holotipo: B十; lectotipo: S!; isolectotipo: HAC!

8. Schmidtottia monticola Borhidi, Acta Bot. Acad. Sci. Hung. 26: 269 (1980). - Tipo: Cuba, Prov. Oriente: Sierra de Moa, charrascos en $800 \mathrm{~m}$ alt. Leg.: Alain (3447), 26.07.1953. Lectotipo: HAC! = Schmidtottia sessiliflora auct. p.p., non Portlandia sessiliflora Britt. 1914, nec Schmidtottia sessiliflora (Britt.) Urb. 1923.

9. Schmidtottia multiflora Urb., Symb. Antill. 9: 141 (1923). - Tipo: Cuba, Prov. Oriente, Sierra Azul in charrascales 500-700 m alt. Leg.: E. L. Ekman (4422). Holotipo: Bt; lectotipo: S!.

10. Schmidtottia neglecta (Borhidi) Borhidi, Acta Bot. Hung. 44: 59 (2002). - Basionym: Schmidtottia shaferi (Standl.) Urb. subsp. neglecta Borhidi, Acta Bot. Acad. Sci. Hung. 26: 272 (1980). $\equiv$ Schmidtottia shaferi sensu Alain p.p., Flora de Cuba 5: 31 (1962), non Standley, N. Am. Fl. 32: 9 (1918). - Tipo: Cuba, Prov. Oriente; pinares cerca de la Mina Franklyn, Moa, alt. 400 m. Leg.: León (23227), 20.07.1947. Holotipo: (LS)HAC!; isotipo: NY!.

11. Schmidtottia nitens (Britt.) Urb., Symb. Antill. 9: 141 (1923). - Basionym: Portlandia nitens Britt., Bull. Torr. Bot. Club 39: 10 (1912). - Tipo: Cuba, Prov. Oriente, Trail Navas to Camp Buena Vista, $650 \mathrm{~m}$ alt., Baracoa. Leg.: A. Shafer (4450), 23.03.1910. Holotipo: NY!; isotipo: HAC!.

12. Schmidtottia parvifolia Alain, Contr. Ocas. Mus. Hist. Nat. Col. "de la Salle" 17: 10 (1959). - Tipo: Cuba, Prov. Oriente, charrascos de la Peña Prieta, La Magdalena, Toa, aprox. 750 m alt. Leg.: Alain (3592), 30.12.1953. Holotipo: HAC!; isotipo NY!.

13. Schmidtottia scabra Borhidi et Acuña, Acta Bot. Acad. Sci. Hung. 17: 30 (1972). - Tipo: Cuba, Prov. Oriente, Moa, Mina Potosí. Leg.: V. Samek (26828), Mayo 1968. Holotipo: HAC!. 
14. Schmidtottia sessilifolia (Britt.) Urb., Symb. Antill. 9: 142 (1923). Basionym: Portlandia sessilifolia Britt., Bull. Torr. Bot. Club 41: 21 (1914). - Tipo: Cuba, Prov. Oriente, Moa, Campo La Gloria, south of Sierra de Moa. Leg.: A. Shafer (8190), 24-30 Dec 1910. Holotipo: NY!; isotipo: HAC!.

15. Schmidtottia shaferi (Standl.) Urb., Symb. Antill. 9: 142 (1923). 72.

15a. Schmidtottia shaferi subsp. shaferi - Basionym: Portlandia shaferi Standl. N. Am. Fl. 32: 9 (1918). = Schmidtottia sessilifolia (Britt.) Urb. subsp. shaferi (Standl.) Borhidi, Bot. Közlem. 58: 176 (1972). - Tipo: Cuba, Prov. Oriente, trail Rio Yamanigüey to Campo Toa, $400 \mathrm{~m}$ alt. Baracoa. Leg.: Shafer (4180), 22-26 Jul 1910. Holotipo: NY!; isotipo: HAC!.

15b. Schmidtottia shaferi subsp. micarensis (Alain) Borhidi, Acta Bot. Hung. 44: 63 (2002). - Basionym: Schmidtottia shaferi (Standl.) Urb. var. micarensis Alain, Contr. Ocas. Mus. Hist. Nat. Col. “de la Salle” 17: 10 (1959). - Tipo: Sierra de Mícara, en la base de la sierra hacia Rio Lebisa. Leg.: Alain y M. López Figueiras, Alain (4833), 30.12.1955. Holotipo: HAC!; isotipos: HAC!, NY!.

16. Schmidtottia stricta Borhidi in Borhidi et al., Abstracta Bot. 5: 38 (1977). - Tipo: Cuba, Prov. Oriente, Moa, Cerro de Miraflores. Leg.: M. Moncada y A. Borhidi (2779711), May 1974. Holotipo: HAC 27797!; isotipo: BP!.

17. Schmidtottia uliginosa (Wernham) Urb., Symb. Antill. 9: 139 (1923). - Basionym: Portlandia uliginosa Wernham, Journ. Bot. London 51: 320 (1913). - Tipo: Cuba, Prov. Oriente, entre Rio Yamanigüey y Campo Toa. Leg.: A. Shafer (4018), Marzo 1910. Holotipo: NY!.

\section{Chiococca y Ramonadoxa}

Paudyal et al. (2018) encontraron en su estudio molecular, que la Chiococca cubensis aparece en un clado fuertemente apoyado y situado como "sister taxon"del género Scolosanthus en una posición muy cercana al clado de Salzmannia y completamente separado del clado del género Chiococca. Esta situación convenció a los autores que es imposible mantener a Chiococca cubensis dentro de los limites del género Chiococca, sino que había que separarlo como un género nuevo monotípico cubano y lo nominaron Ramonadoxa Paludyal et Delprete dedicándolo a Ramona Oviedo-Prieto destacada conocedora y experta en la flora cubana. Según Paudyal et al. las diferencias morfológicas más importantes son, que Ramonadoxa tiene corola 4-mera, tubular-subcilíndrical pardo-rojiza por fuera y amarillo profundo por dentro, lóbulos corolinos estrechamente imbricados, mientras en Chiococca las flores son 5-meras, corola campanulada o embudada blanca, crema o amarilla pálida por fuera y los lóbulos de la corola valvares. 
Destacamos, que debe incluírse en estudios moleculares futuros dos especies endémicas poco conocidas de la flora de Guatemala (C. gracilis Borhidi y C. rubriflora Lundell), que tienen flores 4 y 5 -meras con corola roja por fuera.

\section{CHIOCOCCA P. Browne, 1756}

Chiococca P. Browne, Civ. Nat. Hist. Jamaica, p. 164 (1756). - Tipo: Chiococca alba (L.) Hitchc.

Enredaderas, lianas, arbustos o árboles hermafroditas. Estípulas interpeciolares y parcialmente intrapeciolares, triangulares, persistentes, agudas. Hojas opuestas, sin domácios. Inflorescencias axilares y a veces terminales también, racemosas a paniculadas. Flores con limbo calicino 4 a 5-lobado. Corola infundibuliforme, glabra, lóbulos 5, valvados. Ovario 2-locular, óvulo 1 por lóculo. Fruto drupáceo, carnoso, esponjoso, suborbicular y lateralmente aplanado, blanco con pirenos 2, 1-loculares.

Género neotropical con 25 especies distribuidas desde Texas y Florida hasta Bolivia y Paraguay. 2 en Cuba.

1a Hojas agudas a acuminadas; racimos del largo de las hojas, corola amarilla, tubo de 4 a $8 \mathrm{~mm}$ de largo; estípulas de 2 a 2,5 $\mathrm{mm}$ de largo

1. C. alba

1b Hojas obtusas y más pequeñas, rácimos del largo de las hojas, paucifloros; corola blanca o morada, tubo de 2,5 a $4 \mathrm{~mm}$ de largo, estípulas de 1,5 $\mathrm{mm}$

2. C. parviflora

1. Chiococca alba (L.) Hitchc., Ann. Rep. Miss. Bot. Gard. 4: 94 (1893). - Basionym: Lonicera alba L., Sp. Pl. p. 175 (1753). - Tipo: Jamaica o Barbados fide Howard 1989. Lectotipo: Herb. Sloan, BM!. - Syn.: Chiococca macrocarpa Mart. et Gal., Bull. Acad. Roy. Bruxelles 11: 230 (1844). = Chiococca racemose L., Syst. Nat. ed. 10. 2: 917 (1759), nom. illeg.

2. Chiococca parvifolia Wullschl. ex Griseb., Fl. Brit. W. Ind., p. 337 (1861). - Tipo: Jamaica. Leg.: Wullschlägel s.n. Holotipo probablemente BM n.v.

\section{RAMONADOXA Paudyal et Delprete, 2018}

Tipo: Ramonadoxa cubensis (Urb.) Paudyal et Delprete, Bot. J. Linn. Soc. 187: 25 (2018). Basionym: Chiococca cubensis Urb., Symb. Antill. 9: 163. 1921).

Arbusto $\mathrm{u}$ arbolito de hasta $5 \mathrm{~m}$ de alto; ramas colgantes, cilíndricas, glabras, ramitas ligeramente resinosas; entrenudos basales a veces comprimidas bajo los nudos. Estípulas breves, connadas en la base, persistentes y resinosas. 
Hojas opuestas pecioladas, láminas discoloras, cartáceas a coriáceas, aovada u oblongas. Inflorescencias axilares, paniculadas, pedunculadas , multifloras; brácteas pequeñas, lanceoladas o triangulares. Flores 4-meras, homostilas. Cáliz con tubo estrechamente obovoideo, ondulado o denticulado en el margen. Corola embudada, pardo-rojiza por fuera, amarillo oscuro por dentro, tubo de 10-11 mm de largo, gradualmente ensanchado hacia la garganta, lóbulos cortos, un cuarto del largo del tubo, aovados, obtusos en el ápice, estrechamente imbricados en el botón. Estambres 4, inclusos; filamentos adnatos a la base del tubo corolino, pubescentes en la mitad inferior; anteras lineares. Estilo filiforme, ligeramente engrosado en ápice, más largo que los estambres, ligeramente bilobado. Fruto drupáceo, obovoideo, con dos pirenos leñosos.

El género esta dedicado a Ramona Oviedo-Prieto del Instituto de Ecología y Sistemática, Ministerio de Ciencia, Tecnologia y Medio Ambiente, La Havana, Cuba, actualmente la que mejor conoce la rica y exuberante flora de Cuba.

Ramonadoxa cubensis (Urb.) Paudyal et Delprete, Bot. J. Linn. Soc. 187: 25 (2018)

Basionym: Chiococca cubensis Urb., Symb. Antill. 9: 163 (1921). - Tipo: Cuba. Sierra de Nipe, Río Piedra, in charrascales, 200 m. Leg.: E. L. Ekman (9996), 21 Oct 1919 (fl, fr). Holotipo: Bt; lectotipo: S. - Syn.: Chiococca nitida Griseb., Mem. Amer. Acad. n. ser. 8: 508 (1862), non Benth.

Arbusto $\mathrm{u}$ arbolito de hasta $5 \mathrm{~m}$ de alto escandente, glabro. Ramitas \pm comprimidas debajo de los nudos, la yema apical y los nudos resinosos; las más adultas cilíndricas. Estípulas connadas en un anillo truncado, muy poco apiculadas. Hojas con pecíolos de 7 a $20 \mathrm{~mm}$ de largo, aovadas a oblongas de 6 a $11 \mathrm{~cm}$ de largo y 2.5 a 6,5 cm de ancho, agudas y contraídas en el pecíolo o raramente obtusiúsculas en la base, obtusas o acuminadas en el ápice; nervio medio prominente en el surco del haz, los laterales 5 a 7 pares hundidos en el haz, y angostamente prominentes en el envés, no o muy obsoletamente anastomosados, en arcos conectados en 2 a $3 \mathrm{~mm}$ antes del margen; lámina discolora, nítida en el haz, cartácea a coriácea. Inflorescencias axilares con pedúnculos de 6 a $15 \mathrm{~mm}$ de largo, paniculados por los ramulos inferiores más evolutos, de hasta $5 \mathrm{~cm}$ de largo; brácteas lanceoladas o triangulares de 0,5 a 1 $\mathrm{mm}$ de largo; pedicelos de 1,5 a $2 \mathrm{~mm}$ de largo. Hipanto estrechamente obovado, limbo del cáliz obsoletamente undulado-denticulado. Corola amarilla, por 4 lineas púrpuras decorada, de 10 a $11 \mathrm{~mm}$ de largo; tubo de la corola subcilíndrico arriba y de $3 \mathrm{~mm}$ de ancho, atenuado hacia la base; lóbulos 4, brevemente aovados, obtusos, 4 veces más cortos que el tubo. Estambres 4 , filamentos de $4 \mathrm{~mm}$ de largo, adnatos a la base del tubo corolino y connadas entre sí sobre la base, pubescentes debajo de la mitad; anteras lineares de 3,5 $\mathrm{mm}$ de largo. 
Estilo de $11 \mathrm{~mm}$ de largo, poco engrosado en el ápice, muy brevemente bilobado. Fruto obovado, carnoso, de $7 \mathrm{~mm}$ de largo y $4 \mathrm{~mm}$ de ancho.

Ecol.: Bosque de pinos sobre serpentina en Cuba Oriental (Pinar) y Matorral xeromorfo sub-espinoso sobre serpentina (charrascal), Bosque pluvial sub-montano y Bosque de galería.

Distr.: Cuba: Ho: Mayarí abajo, Sierra de Nipe, Sierra de Moa, Gu: Baracoa. Endémica.

\section{Exostema y Solenandra}

El primer tratamiento sintético de los taxones existentes en Cuba fue publicado en el tomo V. de la Flora de Cuba por Alain en 1962 incluyendo 18 especies. La revisión crítica de esta versión fue publicada por Borhidi y Fernández-Zequeira (1989) que redujo 8 especies a la sinonimia y simultaneamente añadió 9 especies y 3 subespecies nuevas para la ciencia aumentando la lista de las especies válidas a 21. Borhidi catalogó 30 especies antillanas distribuyéndolas en 5 secciones nuevas (1989: 300-301). La monografía taxonómica del género completo está confeccionada por Tim McDowell (1996) quién enriqueció nuestros conocimientos con muchas observaciones y elementos nuevos importantes. En su concepto consideró el género Exostema como una unidad estrictamente monofilética - junto a las especies sudamericanas. Este concepto fue confirmado también por un estudio filogenético molecular detallado (McDowell y Bremer 1998), que dividió el género en 3 secciones (sect. Exostema, sect. Pitonia DC., y sect. Brachyantha DC.), que resultó erróneo, porque utilizaron solamente un solo grupo exterior Coutarea estrechamente asociado a las especies sudamericanas de Exostema mediante $C$. andrei. El estudio molecular de Johan Rova (1999) llamó por primera vez la atención al carácter polifilético posible de Exostema mostrando, que Exostema ixoroides pertenece a un clado separado. Utilizando y ampliando los resultados de los estudios morfológicos y biológicos de McDowell, Borhidi revalidó el género Solenandra Hook. f. con 12 especies basándolo en Solenandra ixoroides como tipo con 5 subespecies y las especies de la sección Brachyantha sensu McDowell. Aunque la validez del género Solenandra ha sido confirmada varias veces por estudios moleculares (Motley et al. 2005, Manns y Bremer 2010), McDowell insistió al mantener su concepto monofilético criticando el restablecimiento de Solenandra por Borhidi (Exostema: por McDowell in Flora Mesoamericana Vol. 4 part 2, 2012: 86). Teniendo en cuenta, que existieron incertidumbres no resueltas, en la Rubiáceas de Cuba (Borhidi, Fernández y Oviedo 2017) aceptamos el concepto amplio del género Exostema del Catálogo de las Indias Occidentales (Acevedo-Rodriguez y Strong 2012) y del World Checklist of Rubiaceae (Govaerts 2016) y tratamos a Solenandra como subgénero de Exostema, aunque 
estuvimos convencidos, que el género Exostema en el sentido amplio de los autores mencionados es polifilético. Acertadamente, los estudios moleculares de Paudyal et al. (2018) produjeron resultados fiables y decisivos. El género Exostema monofilético en sentido de McDowell está separado en 4(!) géneros distintos de la siguiente forma:

- Adolphoduckea Paudyal et Delprete 2018 (basado en Exostema maynense Poepp., vive en Perú);

- Motleyothamnus Paudyal et Delprete 2018 (basado en Portlandia corymbosa Ruiz et Pav. = Exostema corymbosum (Ruiz et Pav.) Spreng., vive en Perú);

- Solenandra Hook. f. em. Paudyal et Delprete 2018 (basado en Solenandra ixoroides Hook. f. = Exostema valenzuelana A. Rich. nom. illeg., vive en las Antillas, México y Mesoamérica);

- Exostema (Pers.) Bonpl. in H. et B. 1807 (basado en Cinchona caribaea Jacq. = Exostema caribaeum (Jacq.) Roem. et Schult., vive (Florida, Bahamas, Antillas, México, América Central).

Notamos, que el nombre sectio Pitonia DC. (Prodr. 4: 359, 1830) está utilizado por McDowell (Exostema sect. Pitonia DC. em. McDowell) y por Paudyal et al. (Solenandra sect. Pitonia (DC.) Paudyal et Delprete) en sentido distinto al contenido y propuesta original de De Candolle. La sectio Pitonia DC. es ilegítima, porque en la descripción original de De Candolle la sección Pitonia incluye en primer lugar a la especie típica del género Exostema, consecuentemente la sectio Pitonia DC. es un sinónimo de la sectio Exostema. Entre las especies listadas en la sección Pitonia DC. se encuentra también la especie típica del género Solenandra, consecuentemente la sectio Pitonia DC. puede considerarse como un sinónimo de la sect. Solenandra como el nombre legítimo. El nombre sect. Brachyanthum DC. (Prodr. 4: 360) tampoco es válido, porque incluye la especie típica del género Motleyothamnus.

Para sustituir el nombre Solenandra sect. Pitonia (DC.) Paudyal et Delprete, 2018. Basionym: Exostema section Pitonia DC., Prodr. 4: 359 (1830); emend. McDowell Opera Bot. Belg. 7: 293 (1996). Lectotipo: Exostema sanctae-luciae (Kentish) Britten (designado por McDowell, Opera Bot. Belg. 7: 293 (1996) proponemos utilizar el nombre válido siguiente:

Solenandra sect. Floribundae (Borhidi) Borhidi, Fernández et Oviedo, comb. nova, hoc loco. Basionym: Exostema sect. Floribundae Borhidi Acta Bot. Hung. 35: 301 (1989). Lectotipo: Exostema sanctae-luciae (Kentish) Britten.

La versión modificada de los géneros arriba discutidos es la siguiente: 


\section{Exostema (Pers.) Bonpl. in H. et B., 1807}

Exostema (Pers.) Bonpl. in Humb. et Bonpl., Pl. Aequin. 1: 131 (1807). - Lectotipo: Exostema caribaeum (Jacq.) Roem. et Schult.

Arbustos y árboles hermafroditas. Estípulas interpeciolares y a veces parcialmente intrapeciolares, triangulares, persistentes. Hojas opuestas o raras veces verticiladas, a veces con domacios. Flores homostilas, generalmente fragantes nocturnas, terminales o axilares, solitarias o en cimas compuestas, brácteadas. Limbo calicino (4) a 5 a (6)-lobulado. Corola hipocrateriforme, blanca, tornándose rosada o morada con la edad; lóbulos (4) a 5 a (6), igual o más largos que el tubo, lineares, fuertemente recurvos. Estambres inclusos en la base de la corola, largamete exertos. Anteras basífijas, lineares. Ovario 2-locular, óvulos numerosos. Fruto una cápsula oblonga, cilíndrica o elíptica, septicida y basípeta, leñosa. Semillas oblongas, aplanadas, con ala membranácea marginal.

Género del Caribe con unas 10 especies, con la mayoría en las Antillas, en Cuba 7 especies.

1a Ramitas espinosas, hojas muy pequeñas de 3-12 $\mathrm{mm}$ de largo

7. E. spinosum

1b Plantas inermes, hojas más grandes

2a Hojas de 7-11 mm de ancho, obtusas en el ápice, sentadas o casí

6. E. salicifolium

2b Hojas agudas o acuminadas en el ápice, pecioladas

3a Hojas membranosas, algo falcadas y mayormente plegadas a lo largo del nervio medio, domaciadas en el envés; flores de 6-10 $\mathrm{cm}$ de largo

1. E. caribaeum

3aa Hojas, peciolos y cáliz glabros

1a. var. caribaeum

3bb Envés de la hoja pubérulo a tomentoso

1b. var. velutinum

3b Hojas coriáceas, no plegadas, sin domacias, flores de hasta $3-4 \mathrm{~cm} \quad 4$

4a Hojas lanceoladas a lineal-lanceoladas o lineal-oblongas, colgantes; peciolo largo y mayormente flexuoso

3. E. lancifolium

4b Hojas aovadas o elípticas a oblongo-elípticas; el peciolo corto y recto 5

5a Margen de las hojas revoluto, el ápice obtuso 5. E. revolutum

5b Hojas del margen plano o poco recurvo, el ápce agudo o acuminado 6 
6a Hojas aovadas de 2,5-3,5 cm de largo, lustrosas en el haz

2. E. glaberrimum

6b Hojas elípticas a lanceoladas de 3,5-9 cm de largo, mayormente mates en ambas caras

4. E. purpureum

6bb Hojas con nervios laterales conspicuos y reticulación aparente, inflorescencias 3-floras 4a. subsp. purpureum

6cc Nervios laterales y nerviación no conspicuos, inflorescencias 1-2-floras

4b. subsp. avenium

1. Exostema caribaeum (Jacq.) Roem. et Schult., Syst. Veg. 5: 19 (1819). - Basionym: Cinchona caribaea Jacq., Enum. Pl. Carib. p. 16 (1760). - Tipo: Dominica: sin localidad precisa. Leg.: De Ponthieu s.n. Lectotipo: BM!. - Syn.: Cinchona jamaicensis Wright, Phil. Trans. Roy. Soc. London, 67: 506 (1778). = Cinchona myrtifolia Stikes, Bot. Mat. Med. 1: 359 (1812). = Cinchona racemosa Schrank, Steud. Nom. Bot. ed. 2. 1: 363 (1840). = Exostema longicuspe Oerst., Vidensk. Meddel Dansk Naturhist. Foren. Kjobenhavn 1852: 48 (1853).

Exostema caribaeum var. caribaeum con hojas, ramitas y pedicelos glabros.

Exostema caribaeum var. velutinum Urb., Symb. Ant. 8: 685 (1921).

2. Exostema glaberrimum Borhidi et Fernández, Acta Bot. Hung. 35: 302 (1989). - Tipo: Cuba Orientalis; prov. Guantánamo, Baracoa; parte alta del Yunque de Baracoa; bosque siempreverde mesófilo de caliza. Leg.: Arias et al., 17.04.1986. Holotipo: HAJB 58878.

3. Exostema lancifolium Borhidi et Acuña in Borhidi et Muñiz, Acta Bot. Hung. 17: 28 (1971). - Tipo: Cuba, Prov. Holguín, Región de Moa, in fruticetis serpentinosis ad Mina Potosí, supra Yamanigüey. Leg.: V. Samek, 1968. Holotipo: SV 26830 HAC.

3a. Exostema lancifolium var. lancifolium

3b Exostema lancifolium var. lucidum (Borhidi et Fernández) Borhidi, Rubiáceas de Cuba, p. 110 (2017). - Basionym: Exostema lucidum Borhidi et Fernández, Acta Bot. Hung. 35: 302 (1989). - Tipo: Cuba Oriental; prov. Holguín; Moa; Barranco del arroyo Jaragua cerca de Minas Jaragua, alt. 300 m s.n.m. Leg.: Álvarez et al., 03.05.1980. Holotipo: HAJB 42659; isotipo: HAJB.

4. Exostema purpureum Griseb., Cat. Pl. Cub. p. 125 (1866). - Tipo: Cuba, Baracoa. Leg.: C. Wright 2761. Holotipo: GOET; isotipos: GH, HAC, NY, BM, S, US.

\section{4a. Exostema purpureum subsp. purpureum}

4b. Exostema purpureum subsp. avenium Borhidi et Fernández, Acta Bot. Hung. 35: 302 (1989). - Tipo: Cuba Orientalis. Leg.: C. Wright 2680. Holotipo: HAC; isotipos: GH, NY, BM, S, US. 
5. Exostema revolutum Borhidi et Fernández, Acta Bot. Hung. 35: 301 (1989). - Tipo: Cuba oriental; Prov. Guantánamo; Laderas al noroeste de la confluencia del Río Baracoa con el arroyo del Cayo; Peladeros de Jauco. Leg.: Berazain et al., 20 Feb. 1979. Holotipo: HAJB 39753; isotipo: HAJB.

6. Exostema salicifolium Griseb., Cat. Pl. Cub. p. 125 (1866). - Tipo: Cuba Occidental. Leg.: C. Wright 2676. Holotipo: GOET; isotipos: GH, HAC!.

7. Exostema spinosum (Le Vavass.) Kr. et Urb. in Urban, Ber. Deutsch. Bot. Ges. 15: 262 (1897). - Basionym: Cinchona spinosa Le Vavass., Obs. Phys. 37: 243 (1790). - Tipo: no designado. - Syn.: Catesbaea vavassorii Spreng., Syst. 1: 116 (1825). = Exostema vavassorii Griseb., Cat. Pl. Cub. p. 126 (1866). = Catesbaea elliptica Spreng. ex DC., Prodr. 4: 401 (1830).

\section{Solenandra Hook. f. 1873 emend. Paudyal et Delprete, 2018}

Solenandra Hook. f. in Benth et Hook. f., Gen. Pl. 2: 43 (1873). - Tipo: Solenandra ixoroides Hook. f.

Frutices o árboles hermafroditas, glabros a pubescentes con ramas cilíndricas. Estípulas interpeciolares pequeñas, caducas. Hojas opuestas, pecioladas a subsésiles, cartáceas a coriáceas. Flores pequeñas, numerosas en corimbos tricotómicamente ramosos terminales, pediceladas, ramas primarias foliosas en la base. Hipanto obovado; cáliz 5-dentado, dientes muy cortos y estrechos. Corola blanca o de color crema, hipocrateriforma, tubo glabro por dentro, mayormente más largo que los 5 lóbulos extendidos, imbricados, cortos, raramente reflejos. Estambres 5, filamentos insertos en la base del tubo corolino, filamentos connados en un tubo hasta más de la mitad, libres arriba, exertos, anteras basifijas, lineares, obtusas. Disco inconspicuo, estilo entero, capitado; ovario 2-locular, óvulos pocos, pendulos. Cápsula obovoide septicida, comprimida, 2-valva. Semillas pocas, imbricadas, comprimidas, aladas.

Género antillano con 22 especies, con una especie en México y América Central y 13 en Cuba.

1a Flores con corolas de 4 a $21 \mathrm{~cm}$ largo, blancas durante la floración, cambiando el color después, a rosadas, rojas oscuras o violáceas, fragantes por la noche (sect. Floribundae)

1b Flores con corolas de 1-3 cm de largo, blancas durante la floración luego pálidamente flavescentes, fragantes por el día (sect. Solenandra) 5

2a Hojas lineales a lineal-lanceoladas, flor de 9-15 cm de largo 3

2b Hojas elípticas, aovadas u obovadas, flor de $4-8 \mathrm{~cm}$ de largo 4 
3a Hojas membranosas, estrechadas a agudas en el ápice, ramitas jóvenes pubérulas a vellosas, flores mayormente axilares

6. S. longiflora

3b Hojas subcoriáceas a coriáceas, obtusas a redondeadas en el ápice, ramitas glabras, flores terminales

12. S. stenophylla

4a Hojas obovadas a suborbiculares, tubo del cáliz anguloso, tubo de la corola el doble del largo de los lóbulos

10. S. rotundata

4b Hojas elípticas o aovadas, mayormente acuminadas o agudas, tubo del cáliz no anguloso, tubo de la corola de igual largo o más corto que los lóbulos

4. S. elliptica

5a Arbustos micrófilos con hojas coriáceas de hasta 3(-4) $\mathrm{cm}$ de largo 6

$5 \mathrm{~b}$ Arbustos y árboles con hojas más grandes

6a Hojas sin domacio, pelosas en las axilas de los nervios del envés $\quad 7$

6b Hojas comúnmente con domacio

7a Hojas anchamente acorazonadas de hasta $4 \mathrm{~cm}$ de largo, brevemente pubérulas en ambas caras

1. S. cordata

$7 \mathrm{~b}$ Hojas aovadas o elípticas a suborbiculares, más pequeňas

8a Hojas de hasta $3,5 \mathrm{~cm}$, pelosas en los nervios del envés; fruto pequeňo, subgloboso de hasta $3 \mathrm{~mm}$ de largo

6. S. microcarpa

8b Hojas de hasta 2,5 cm; glabras y nitidas en ambas caras, fruto de 6-7 $\mathrm{mm}$

1. S. curbeloi

9a Hojas elípticas a lanceoladas de 2-3,5 cm de largo, fuertemente domaciadas y brevemente pelosas en el envés, nervios laterales $4-5$ pares, pecíolo de 2,5-4 cm de largo

4d. S. ixoroides subsp. parvifolia

9b Hojas aovadas a suborbiculares o deltoideas de 0,7-1,8 cm de largo, subsésiles, nervios laterales $2-3$ pares

10a Hojas escabrosas en el haz con pelos tuberculados en la base

11. $S$. scabra

10b Hojas lisas a plegaditas en el haz sin pelos o tubérculos 7 . S. myrtifolia 10aa Hojas glabras, mayormente sin domácio

7a. var. myrtifolia 10bbHojas vellosas en el nervio medio del envés, mayormente con domacio

$7 \mathrm{~b}$. var. barbata 
11a Hojas no aterciopeladas a pulverulentas en el envés 4. S. ixoroides

11b Hojas densamente pulverulentas o aterciopeladas en el envés 15

12a Hojas aovadas de 5-10 cm de largo, domácias escasas o ausentes, nerviación aparente en el envés

4b. subsp. eggersii

12b Hojas de 3-6 cm, mayormente domaciadas

13a Hojas elípticas a oblongo-aovadas, atenuadas en la base membranosas a cartáceas, domacio en las axilas superiores del envés

4c. subsp. maestrensis

13b Hojas aovadas, obtusas redondeadas a subacorazonadas en la base, subcoriáceas a coriáceas

14a Cáliz glabro a glabrescente, hojas obtusas a redondeadas en la base, con pelos largos esparcidos en los nervios del envés 4a. subsp. ixoroides

14b Cáliz pubérulo a densamente pubescente, hojas redondeadas truncadas a subacorazonadas en la base, poco abolladas, nervios fuertemente impresos en el haz, corto pubérulos en el envés

4e. subsp. wrightii

15a Hojas lampiňas en el haz

13. S. velutina

15b Hojas pulverulentas o aterciopeladas en ambas caras

16a Hojas oblongo-elípticas a lanceoladas, pulverulentas en ambas caras

9. S. pulverulenta

16b Hojas anchamente aovadas, aterciopeldas en ambas caras

8. S. pervestita

1. Solenandra cordata (Borhidi et Fernández) Borhidi, Acta Bot. Hung. 44: 227 (2002). - Basionym: Exostema cordatum Borhidi et Fernández, Acta Bot. Hung. 35: 303 (1989). - Tipo: Cuba: prov. Oriente, Sardinero al Sur de Santiago

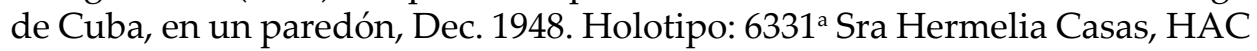
(LS); isotipo: $6331^{\mathrm{b}} \mathrm{Hno}$ Clemente HAC(LS).

2. Solenandra curbeloi (Borhidi et Fernández) Borhidi, Acta Bot. Hung. 44: 227 (2002). - Basionym: Exostema curbeloi Borhidi et Fernández, Acta Bot. Hung. 35: 304 (1989). - Tipo: Cuba. Prov. Oriente, Puerto Padre, orillas de arroyos. Leg.: Curbelo (105), 8 Dec 1931. Holotipo: Herb. Roig 5857 HAC; isotipo: HAC.

3. Solenandra elliptica (Griseb.) Paudyal et Delprete, Bot. J. Linn. Soc. XX: 24 (2018). - Basionym: Exostema ellipticum Griseb., Pl. Wright. 2: 504 (1862). Tipo: Cuba. Oriente: Guantanamo Prov., 'in sylvis prope Monte Verde'. Leg.: C. Wright (1257), Jan-Jul 1859 (fl, fr). Lectotipo: GOET; isolectotipos: BR, K, 
MO, NY, PH, S, US, YU. - Syn.: Exostema monticola Borhidi et Fernández, Acta Bot. Hung. 35: 303 (1989). - Tipo: Cuba; Prov. Oriente, Sierra Maestra, Loma Pino de Agua, Alto Valenzuela, 1200 a 1400 m snm. Leg.: López Figueiras L. F. (2314), 11.08.1955. Holotipo: HAC; isotipos: HAC, HAJB.

\section{3a. Solenandra elliptica subsp. ellipticum}

3b. Solenandra elliptica subsp. monticola (Borhidi et Fernández) Borhidi et Fernández, comb nova, hoc loco - Basionym: Exostema monticola Borhidi et Fernández, Acta Bot. Hung. 35: 303 (1989). = Exostema ellipticum Griseb. subsp. monticola Borhidi et Fernández, Rubiáceas de Cuba, p. 108 (2017). - Tipo: Cuba, Prov. Oriente, Sierra Maestra, Loma Pino de Agua, Alto Valenzuela, 1200 a 1400 m snm. Leg.: López Figueiras L. F. (2314), 11.08.1955. Holotipo: HAC; isotipos: HAC, HAJB.

4. Solenandra ixoroides Hook. f. in Hook. f., Gen. Pl. 2: 43 (1873). =Exostema ixoroides (Hook. f.) McDowell, Bissea 3 (num. esp.): 82 (2009). - Tipo: dibujo de W. H. Fitch in Hooker J. D., Icones Plantarum 1876. - Syn.: Exostema parviflorum A. Rich., Sagra Hist. Nat. Cuba X, p. 14 (1845), non E. parviflorum

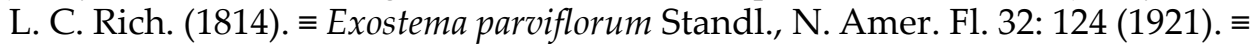
Exostema parviflorum Alain, Fl. Cuba 5: 25 (1962), non L. C. Rich. (1808). = Exostema valenzuelae A. Rich., Sagra Hist. Nat. Cuba XI, plate 48, (1850), sine descriptione, nom. illeg. = Exostema wrightii Kr. et Urb., Symb. Ant. 1: 424 (1899). = Exostema eggersii Urb., Symb. Ant. 9: 521 (1928). = Exostema elegans Alain, Fl. Cuba 5: 25 (1962), non Kr. et Urb. (1899).

4a. Solenandra ixoroides subsp. ixoroides - Syn.: Exostema valenzuelae A. Rich. subsp. valenzuelae Borhidi et Fernandez, Acta Bot. Hung. 35: 305 (1989). - Tipo: Cuba. Prov. Pinar del Río, Valenzuela, s.n. Holotipo: P!. Descriptio sub nom. E. parviflorum A. Rich. in Sagra, Hist. Nat. Cub. X: 14 (1845). इ E. parviflorum auct. non L. C. Rich. ex Bonpl. in Humboldt et Bonpland., Pl. Aequinoct. 1: 132 (1808), nec E. elegans Kr. et Urb., Symb. Ant. 1: 423 (1899).

4b. Solenandra ixoroides subsp. eggersii (Urb.) Borhidi, Acta Bot. Hung. 44: 227 (2002). - Basionym: Exostema eggersii Urb., Symb. Ant. 9: 521 (1928). 三 Exostema parviflorum Rich. subsp. eggersii (Urb.) Borhidi, Bot. Közlem. 61: 157 (1973). = Solenandra ixoroides Hook. f. subsp. eggersii (Urb.) Borhidi, Acta Bot. Hung. 44: 227 (2002). E Exostema ixoroides McDowell subsp. eggersii (Urb.) Greuter, Bissea 3 (num. esp.): 82 (2009). - Tipo: Cuba, prov. Oriente, prope El Palenquito, in sylvestribus $600 \mathrm{~m}$ alt. Leg.: Eggers (4809), Mart. fruct. Holotipo: B十; lectotipo: Cuba, Prov. Oriente, Mesa de Prada, Jauco. Leg.: León (11958), 17 Jul-4 Aug 1924, HAC(LS); isolectotipos: NY, US. - Syn.: Exostema parviflorum Standl., N. Amer. Fl. 32: 124 (1921), non E. parviflorum Rich. (1814).

4c. Solenandra ixoroides subsp. maestrensis Borhidi et Fernández, Acta Bot. Hung. 44: 227 (2002). $\equiv$ Solenandra ixoroides Hook. f. subsp. maestrensis (Borhidi et Fernández) Borhidi, Acta Bot. Hung. 44: 227 (2002). 三 Exostema 
ixoroides McDowell subsp. maestrensis (Borhidi et Fernández) Greuter, Bissea 3 (num. esp.): 82 (2009). - Tipo: Cuba, Prov. Oriente, bordes del Río Portillo, Sierra Maestra, Pico Turquino. Leg.: J. Acuña (10218), 26.10.1936. Holotipo: HAC (LS); isotipo: Herb. Roig, 7742 HAC. - Syn.: Exostema elegans Alain, Fl. Cuba 5: 25 (1962), p.p. non E. elegans Kr. et Urb. (1899).

4d. Solenandra ixoroides subsp. parvifolium Borhidi et Fernández, Acta Bot. Hung. 44: 227 (2002). = Solenandra ixoroides subsp. parvifolia (Borhidi et Fernández) Borhidi, Acta Bot. Hung. 44: 227 (2002). 三 Exostema ixoroides McDowell subsp. parvifolia (Borhidi et Fernández) Greuter, Bissea 3 (num. esp.): 82 (2009). - Tipo: Cuba, Prov. Oriente, Palenque Cuchillas de Toa, Cayo Fortuna, pinares y charrascos en el trillo de Riíto a Piloto Arriba. Leg.: J. Bisse y R. Berazain, Apr. 1972. Holotipo: HAJB 22258.

4e. Solenandra ixoroides subsp. wrightii (Kr. et Urb.) Borhidi, Acta Bot. Hung. 44: 227 (2002). - Basionym: Exostema wrightii Kr. et Urb. in Urb., Symb. Ant. 1: 424 (1899). = Exostema parviflorum Rich. subsp. wrightii (Kr. et Urb.) Borhidi, Bot. Közlem. 61: 157 (1973). इ Solenandra ixoroides Hook. f. subsp. wrightii (Kr. et Urb.) Borhidi, Acta Bot. Hung. 44: 227 (2002). इ Exostema ixoroides McDowell subsp. wrightii (Kr. et Urb.) Greuter, Bissea 3 (num. esp.): 82 (2009). - Tipo: Cuba orientalis, Monte Verde, C. Wright 2672. Holotipo: GOET; isotipos: GH, HAC. - Syn.: Exostema parviflorum Standl., N. Amer. Fl. 32: 124 (1921) p.p. $\equiv$ Exostema parviflorum Alain, Fl. Cuba 5: 25 (1962) p.p.

5. Solenandra longiflora (Lamb.) Paudyal et Delprete, Bot. J. Linn. Soc. XX: 24 (2018). - Basionym: Cinchona longiflora Lamb., Descr. Cinchona 38, figure 12 (1797). इSyn.: Exostema longiflorum (Lamb.) Roem. et Schult., Syst. Veg. 5: 18 (1819). - Tipo: Haiti, without locality, s.d. [1764-1765], J. B. C. Aublet s.n. Lectotipo: BM.

6. Solenandra microcarpa (Borhidi et Fernández) Borhidi, Acta Bot. Hung. 44: 227 (2002). - Basionym: Exostema microcarpum Borhidi et Fernández, Acta Bot. Hung. 35: 304 (1989). - Tipo: Cuba, prov. Oriente, Santiago de Cuba, Cerca de la embocadura del Río San Juan, Playa de Aguadores. Leg.: L. F. (718), 26 Oct 1952. Holotipo: HAC; isotipos: HAC, HAJB, NY.

7. Solenandra myrtifolia (Griseb.) Borhidi, Acta Bot. Hung. 44: 227 (2002). - Basionym: Exostema myrtifolium Griseb., Cat. Pl. Cuba p. 125 (1866). - Tipo: Cuba, prov. Oriente, Cuchillas de Baracoa. Leg.: C. Wright (2673). Holotipo: GOET; isotipos: GH, HAC!. - Syn: Exostema barbatum Standl., N. Amer. Fl. 32: 125 (1921). = Exostema crassifolium Standl., N. Amer. Fl. 32: 124 (1921). = Exostema shaferi Standl., N. Amer. Fl. 32: 124 (1921). = Exostema nipense Urb., Symb. Ant. 9: 521 (1928). = Exostema dumosum Alain, Contr. Ocas. Col. De La Salle, La Habana 17: 3 (1959).

7a. Solenandra myrtifolia var. myrtifolia

7b. Solenandra myrtifolia var. barbata (Standl.) Borhidi, Acta Bot. Hung. 44: 227 (2002). - Basionym: Exostema barbatum Standl., N. Amer. Fl. 32: 125 
(1921). - Tipo: Cuba, Pine hills near Baracoa, Oriente. Leg.: L. M. Underwood y F. S. Earle (1360), March 1903. Holotipo: NY.

8. Solenandra pervestita (Borhidi et Fernández) Borhidi, Acta Bot. Hung. 44: 230 (2002). - Basionym: Exostema pervestitum Borhidi et Fernández, Acta Bot. Hung. 35: 307 (1989). - Tipo: Cuba, prov. Oriente, vertientes de Maisí. Leg.: Hno León y Matos, Apr. 1939. Holotipo: HAC(LS); isotipo: NY.

9. Solenandra pulverulenta (Borhidi) Borhidi, Acta Bot. Hung. 44: 230 (2002). - Basionym: Exostema pulverulentum Borhidi, Acta Bot. Hung. 37: 79 (1992). - Tipo: Cuba, prov. Santa Clara, Trinidad Mountains, San José, on low cliffs. Leg.: L. B. Smith, A. R. Hogdon y F. Gonzalez (3268), 29 Jul 1936. Holotipo: NY; isotipos: GH, JPU.

10. Solenandra rotundata (Griseb.) Paudyal et Delprete, Bot. J. Linn. Soc. XX: 24 (2018). - Basionym: Exostema rotundatum Griseb., Pl. Wright. 2: Mem. Acad. Amer. N. S. 8: 504 (1862). E Exostema ellipticum var. rotundatum (Griseb.) Maza, Anales Soc. Esp. Hist. Nat. 23: 286 (1894). - Tipo: Cuba, Oriente: Guantánamo Prov., 'prope villam Monte Verde'. Leg.: C. Wright (1258), Jan-Jul 1859 (fl, fr). Lectotipo: GOET; isolectotipos: BR, GH, HAC, K, MO, PH, YU. - Syn.: Exostema obovatum Alain, Contr. Ocas. Mus. Hist. Nat. "La Salle" 17: 2 (1959).

11. Solenandra scabra (Borhidi et Fernández) Borhidi, Acta Bot. Hung. 44: 230 (2002). - Basionym.: Exostema scabrum Borhidi et Fernández, Acta Bot. Hung. 35: 304 (1989). - Tipo: Cuba, prov. Guantánamo, San Antonio del Sur, Abra Mariana, monte seco, suelo calizo, 100 a $300 \mathrm{~m}$ snm. Leg.: J. Bisse et al. (48177), 21 May 1982. Holotipo: HAJB.

12. Solenandra stenophylla (Torrey) Paudyal et Delprete, Bot. J. Linn Soc. XX: 25 (2018). - Basionym: Exostema stenophyllum Torrey, Bull. Torr. Bot. Club 42: 517 (1915). - Tipo: Cuba, Oriente, Río Guayabo, among rocks at the water's edge, 450-550 m. Leg.: J. A. Shafer (3623), 21 Jan 1920 (fr). Holotipo: NY; isotipos: F, MO, US.

13. Solenandra velutina (Standl.) Borhidi, Acta Bot. Hung. 44: 230 (2002). Basionym: Exostema velutinum Standl., N. Amer. Fl. 32: 125 (1921). - Tipo: Cuba, prov. Santa Clara, (Cienfuegos), at border of mangrove swamp Río San Juan. Leg.: N. L. Britton, F. S. Earle y P. Wilson (5837), March 1910. Holotipo: NY.

\section{REFERENCIAS}

Alain, H. Liogier (1962): Rubiaceae. - In: Flora de Cuba V. Univ. Puerto Rico, Rio Piedras, pp. 13-148.

Borhidi, A. (2002): Los géneros Ceuthocarpus Aiello y Schmidtottia Urb. (Rubiaceae). Acta Bot. Hung. 44: 49-65. https://doi.org/10.1556/ABot.44.2002.1-2.4

Borhidi, A. (2002): Revalidación del género Solenandra Hook. f. (Rubiaceae). - Acta Bot. Hung. 44: 223-231. https://doi.org/10.1556/abot.44.2002.3-4.3

Borhidi, A. (2003): Lorencea gen nov. (Condamineae, Rubiaceae) de Guatemala y México. - Acta Bot. Hung. 45: 13-21. https://doi.org/10.1556/abot.45.2003.1-2.2 
Borhidi, A. y Fernández-Zequeira, M. (1989): El género Exostema L. C. Rich. (Rubiaceae) en Cuba. - Acta Bot. Hung. 35: 287-307.

Borhidi, A., Fernández-Zequeira, M. y Oviedo-Prieto, R. (2017): Rubiáceas de Cuba. - Akadémiai Kiadó, Budapest, 494 pp.

Bremer, B. y Eriksson, T. (2009): Time tree of Rubiaceae phylogeny and dating the family, subfamilies, and tribes. - Int. J. Plant Sci. 170: 766-793. https://doi.org/10.1086/599077

Govaerts, R. (2016): World checklist of Rubiaceae. - Royal Bot. Gard., Kew. http://apps.kew. org./wcsp.

Manns, U. y Bremer, B. (2010): Towards a better understanding of intertribal relationships and stable tribal delimitations within Cinchonoideae s.s. (Rubiaceae). - Mol. Phyl. Evol. 56: 21-39. https://doi.org/10.1016/j.ympev.2010.04.002

McDowell, T. (1996): Exostema (Rubiaceae): taxonomic history, nomenclature, position and subgeneric classification. Proc. 2nd Int. Symp. Rubiaceae - Opera Bot. Belg. 7: 277-295.

McDowell, T. (2012): Exostema (Pers.) Rich. - In: Davidse, G., Sousa, M. S., Knapp, S. y Chiang, F. (eds): Flora Mesoamericana, Vol. 4. parte 2, pp. 86-87.

McDowell, T. y Bremer, B. (1998): Phylogeny, diversity and distribution in Exostema (Rubiaceae): implications of morphological and molecular analyses. - Pl. Syst. Evol. 212: 215-246. https://doi.org/10.1007/bf01089740

McDowell, T., Volovsek, M. y Manos, P. (2003): Biogeography of Exostema (Rubiaceae) in the Caribbean Region in light of molecular phylogenetic analysis. - Syst. Bot. 28 : 431-441.

Motley, T. J., Wurdack, K. J. y Delprete, P. G. (2005): Molecular systematics of the Catesbaeeae-Chiococceae complex (Rubiaceae): flower and fruit evolution and biogeographic implications. - Amer. J. Bot. 92: 316-329. https://doi.org/10.3732/ajb.92.2.316

Paudyal, S. K., Delprete, P. G., Neupane S. y Motley, T. J. † (2018): Molecular phylogenetic analysis and generic delimitations in tribe Chiococceae (Cinchonoideae, Rubiaceae). - Bot. J. Linn. Soc. 187: 365-396. https://doi.org/10.1093/botlinnean/boy029

Rova, J. H. E. (1999): Rubiaceae phylogeny based on rps16 sequence data. - Diss. Bot. Inst. Göteborg Univ. Suppl. 2: 1-29. 